\title{
Transmission Line Fault Analysis Using Artificial Neural Network \\ ${ }^{* 1}$ Arpitsingh Sandhu, ${ }^{2}$ Dr.Vinay Barhate, ${ }^{3}$ Sudarshan Khond \\ ${ }^{1}$ Alumni, Businessman, Khamgaon \\ ${ }^{2,3}$ Shri Ramdeobaba College of Engineering and Management, Nagpur \\ Email: ${ }^{1}$ arpit171294@gmail.com, ${ }^{2}$ barhatevt@rknec.edu, ${ }^{3}$ khondsr1@rknec.edu
}

Received: 06th October 2019, Accepted: 20th November 2019, Published: 31st December 2019

\begin{abstract}
Work presented in this paper elaborates use of Artificial Neural Network (ANN) for detection, classification and location of faults in an interconnected power system. Transmission lines chunks of power to the required loads; it is an essential link between consumers and power stations. Hence transmission lines protection has become critical. A different fault classification technique is proposed in this paper, which yields high accuracy in fault classification with decreased training time and dimension of ANN. The three phase currents and voltages of an end are taken as input to the proposed system. All possible types of faults at various locations were considered. The variation of fault resistance is also considered. The collected data is then used to train the ANN, to locate the fault. The network is modelled and simulated in the MATLAB/Simulink environment. It is apparent in the simulation results obtained that proposed algorithm has satisfactory performance to achieve desired goal of fault identification, classification and determining location.
\end{abstract}

Keywords

Artificial Neural Networks, Back Propagation Algorithm, Fault Classifier, Fault Detector, Fault Locator, Transmission Line Faults.

\section{Introduction}

An overhead electric power transmission line is an essential link between the generating plants and different substations. Transmission lines exclusively responsible for conveying electrical power from different generating plants to the grid and from the grid to different substations and from one substation to another substation in a varying degree of voltages in order to meet the extremely large number of load demands. Thus, Overhead power transmission lines are commonly found to be subjected to faults as they carry power over long distances in vividly extreme environmental conditions. [9]

Depending on the duration, faults can be segregated into permanent and temporary/transient. Although a transient fault is self-cleared by the line by auto reclosing after a few cycles, it is important to determine fault condition and the location of the same in the transmission line. In the case of a permanent fault that persists even after 2-3 auto recloses, control engineers are equipped to determine fault location enabling maintenance crew to efficiently and quickly take necessary measures. Thus, for optimal utilization of electric power generated and reliability of electric power supplied, it is highly desirable and advantageous to detect, classify, locate and clear these faults at the fastest speed. The artificial neural network is a promising online and offline approach to achieving speedy fault detection, classification, and location in overhead transmission lines. Thus this paper aims to develop a fault analysing algorithm that uses an artificial neural network (ANN) which will detect, classify and locate a fault as it occurs.

ANN is found to be a powerful tool for pattern recognition, classification, generalization and it can be used for power system applications owing to its ability to be trained with off-line data. [3] ANN also comes in handy with features such as noise immunity, robustness and fault tolerance. Work in this paper emphasizes on detection, classification and location of faults in an electric power transmission line. ANN algorithm is used for fault detection, classification and location. Neural network analysis with different number of hidden layers and neurons per hidden layer has been done to optimize its requirement.[7] The training testing and evaluation of the intelligent locator is done based on a multilayer perceptron feed forward artificial neural network with backpropagation algorithm. The performance of the detector and classifier was evaluated using Mean Square Error (MSE) and confusion matrix and each locator's performance was evaluated using regression plot and error histogram.

The developed system is capable of detecting and classifying various faults such as single line-to-ground faults, double line-to-ground faults, line-to-line faults, triple line faults and no fault condition as well as to locate the same faults. A Simulink model of transmission line in MATLAB simpower system is developed for training and testing of the ANN by using Neural Network Toolbox in MATLAB.

\section{Power System Model}

The proposed strategy using ANN networks is implemented for $220 \mathrm{kV}$ transmission line. A single line diagram of the system as shown in Figure 1(a) is taken as a reference for further analysis. System under consideration for the simulation has two $250 \mathrm{kV}$ generators both at sending and receiving ends of the pi 
modelled transmission line. The Transmission line has been modelled using six $\Pi$ section of $30 \mathrm{~km}$ length together comprising of $180 \mathrm{~km}$ line length to model a significantly long transmission line for aimed analysis.

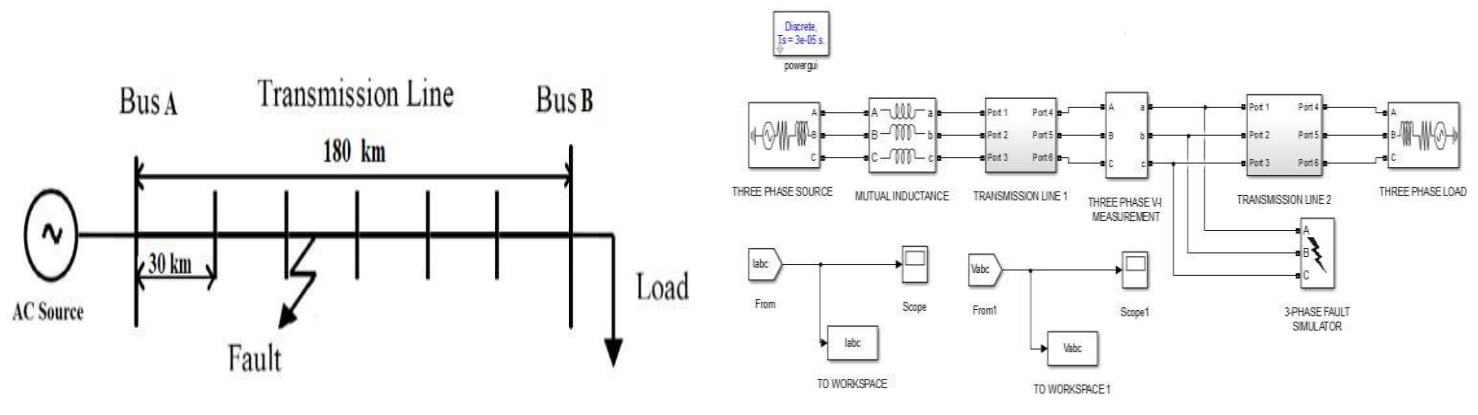

Figure 1: (a) Single Line Diagram of the Studied System

Figure 1: (b) Snapshot of the Model Studied in MATLAB

\section{Simulation Model}

Figure 1(a) shows single line diagram of the system under consideration for classification and location of fault. Figure 1(b) shows screenshot of the MATLAB Simulink model of the same system. ZP is the source impedances on the generators side.

The transmission line (line 1 and line 2 together) is $180 \mathrm{~km}$ long and the fault simulator is deployed to simulate various types and degrees of faults at varying locations along the transmission line by changing type of fault and amount of fault impedance. The required voltage and current samples for ANN training are taken with the help of V-I measurement block at the terminal A. The values of the three-phase voltages and currents are measured and processed accordingly (scaled with respect to their pre-fault values) and are fed in to the neural network unit as inputs for further processing. The SimPower Systems library is used to obtain all the training data for both fault and non-fault conditions.

Data Pre-Processing

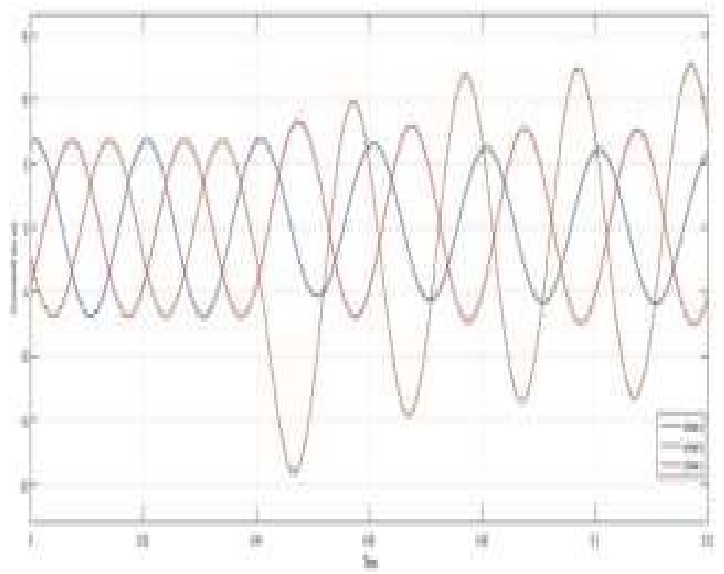

Figure 2: Data Pre-processing Illustration

A size reduction of the neural network results in improved performance of the same which can be achieved by performing pre-processing of data. [6] Figure 2 shows the waveform for current in a L-G fault on phase B at a distance of $60 \mathrm{~km}$ from terminal $\mathrm{A}$ in a $180 \mathrm{~km}$ transmission line. The sampling frequency of the waveform is taken as $1000 \mathrm{~Hz}$.

Hence there are 20 samples for each cycle of a standard $50 \mathrm{~Hz}$ frequency signal. Now, two samples are considered - i)Sample corresponding to post fault condition $\left(60^{\text {th }}\right.$ sample on phase B) and ii)Sample before the occurrence of the fault referred as pre-fault sample. Once these samples are collected, ratio of the voltage and current corresponding to the pre-fault and post fault instances are presented to the ANN as the input.

\section{Methodology}

Two separate ANNs are considered for fault classifier which one is for faults involving earth and another for faults excluding earth resulting in decreased of training time and dimension of each ANN [2] 


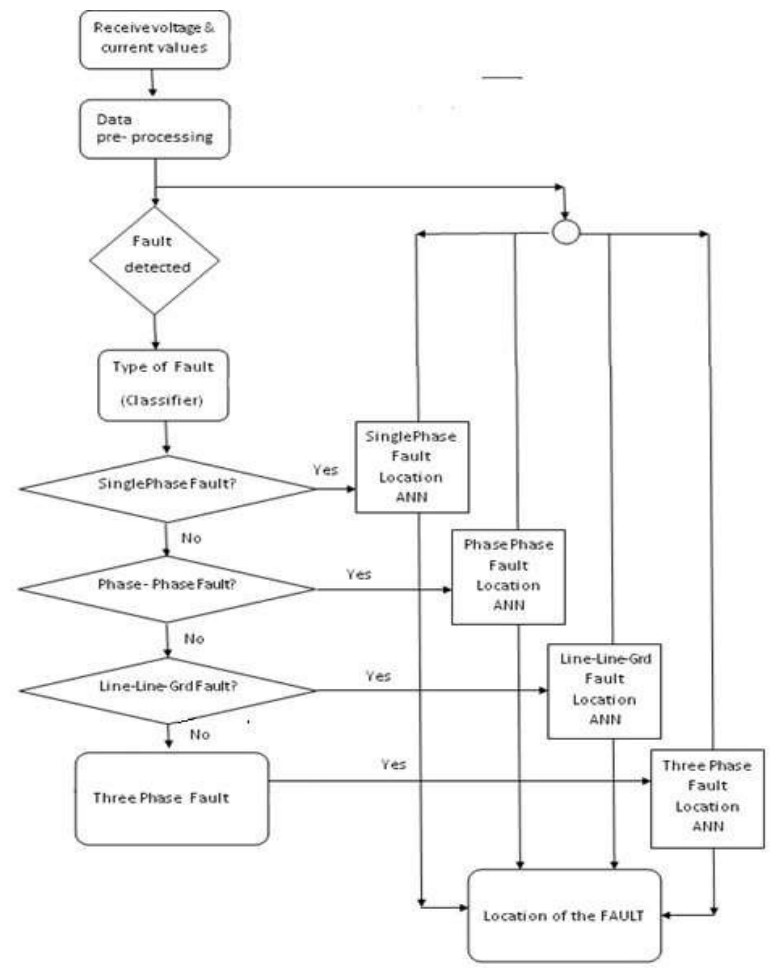

Figure 3: Flowchart of Proposed Work

Signal $G$ explains the existence of faults involving earth and causes to activate the appropriate ANN. For example if $\mathrm{G}=1$, only ANN-I will be activated and the inputs switch to ANN-I. Obviously, in this condition ANN-II has been deactivated and all of its outputs are zero. Hence, ANN-1 will differentiate between LG and LLG fault while ANN-II will differentiate between LL and LLL fault. (Figure. 4(a))

The I0 is the magnitude of zero sequence components of currents. It is used for detection of faults which involving earth. The I0 enters a level detector, and its output is a logical signal (G). By this way, if earth fault happen $\mathrm{G}=1$ otherwise $\mathrm{G}=0$

The fault classification plays an important role in modular neural network based fault location. The overall scheme of proposed ANN- based fault locator is shown in figure.5. It consists of four ANNs to four fault types. The (L-G), (L-L), (L-L-G)and (L-L-L) signals will come from the fault classifier and depending upon the occurred fault, the relative neural network will activate. This would result in decreasing of training time and dimension of each ANN. (Figure 4(b)).

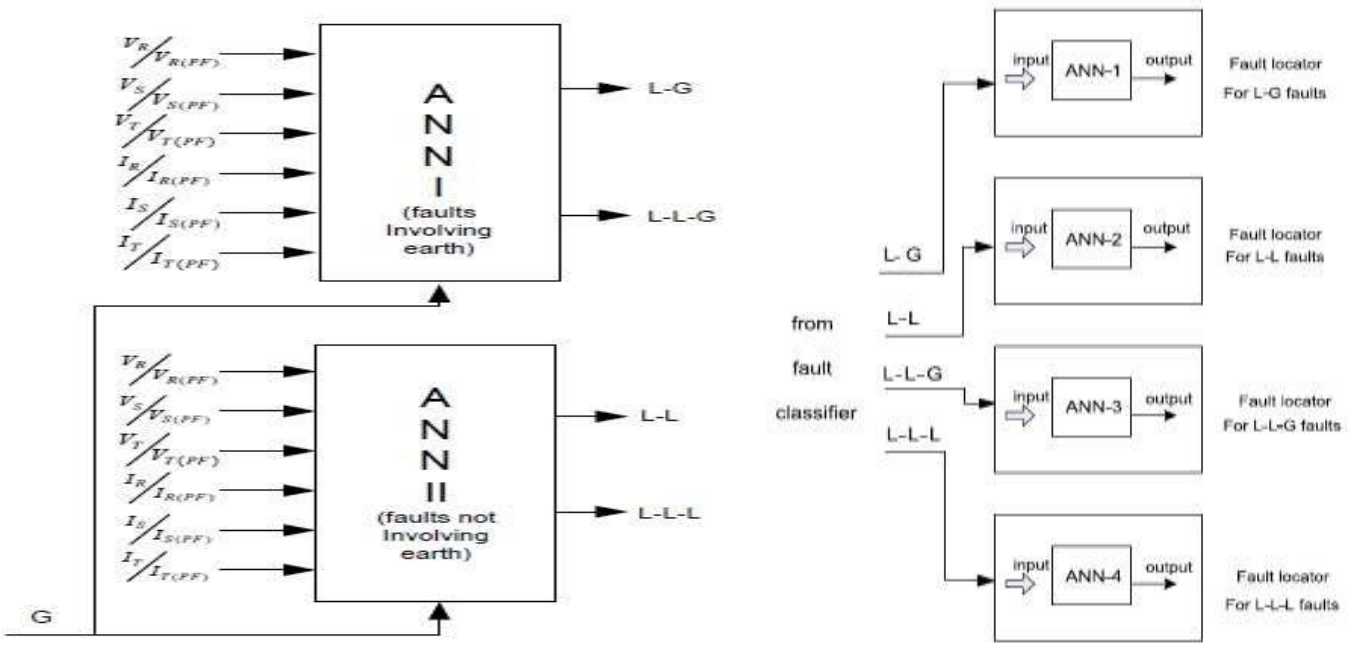

Figure 4: (a) Proposed Fault Classifier Structure Figure 4: (b) Proposed Fault Locator Structure 


\section{Training and Testing of ANN \\ Training:}

Training pertains to the process by means of which the neural network learns from the inputs and updates its weights accordance to achieve target as supervised. A set of input-output pairs fed into the neural network which is termed as training data set in order to train neural networks. With these means, we specify the neural network about what the output should be corresponding to the particular input presented to it. The ANN thus graduates into learning the training set and slowly develops an ability generate similar outputs corresponding to the data received by it during training.

To achieve above, the neural network's weights are updated subject to minimizing the performance function. This performance function can be user defined to meet custom requirements but normally in practice, feedforward networks with backpropogation algorithm use Mean Square Error as the performance function and the same is considered throughout this work.[5]

\section{Testing:}

Testing the artificial neural network is referred to the process in which a trained network is presented with the new data set as input and it is checked whether desired outputs are obtained or not. There are various methods for evaluating performance of a trained ANN, One such technique is to plot the best linear regression fit. This technique defines correlation coefficient (r) of the outputs and the targets and depending on its value it finds how well the ANN outputs track the desired target. Deviation of the value of $r$ from 1 should be minimum for better performance.[5]

Another method to test an ANN for the classification of data is the plot of matrix that determines ability of the network to classify each input successfully. Such matrix is termed as confusion Matrix. An Ideal \% value of confusion matrix is 100 which imply that ANN can clearly classify given set of data. Error histogram plot can also be used to test the network. Difference between target and output is shown in an error histogram. Histogram provides the information regarding data which does not fit into the required set of data to be classified and it also gives extent of the given data's exclusiveness. The final stage of ANN testing corresponds to the presentation of the data set along with the targets. Percentage error is calculated depending on the deviation of the obtained output from the specified targets. If the average percentage error in the $\mathrm{ANN}^{\text {ee }} \mathrm{S}$ output is acceptable as per the threshold set by the user, the neural network is said to have passed the test and can be used to classify future inputs. [4]

\section{ANN Design}

\section{ANN Design for Fault Detection}

ANN is provided with 6 inputs for the detection of the fault. Six inputs correspond to $3 \varphi$ current and the voltage respectively. Input current and voltage values given to the ANN as input are scaled with respect to pre-fault values of voltages \& currents correspondingly. ANN provides output as 1 for occurance of the fault and 0 otherwise. All ten types of fault along with the no-fault situation have been simulated and the fault resistance is also varied to include several possible practical situations. [8]

- The fault resistance has been changed as follows: $0.5,1,2$ and $5 \mathrm{ohms}$

- Fault distance has been changed in an incremental amount of every $30 \mathrm{~km}$ on a $180 \mathrm{~km}$ transmission line

After simulating and observing various network, the selected network to optimize training performance in terms of time and accuracy ANN has input layer with 6 nuerons, a hidden layer containing 10 neurons in it with 1 neuron in output layer. Purlin and tansig are considered as transfer functions in hidden and output layers.

From the training performance plot as shown in following figures, it can be observed that training operation shown by ANN is reasonable. MSE value of 3.9428e-7 is delivered in completion of training ANN. To index the performance of ANN, confusion matrices are plotted for varieties of inaccuracies occurred for trained ANN.

\section{ANN Design for Fault Classification}

Fault classifier has been designed as by the scheme proposed earlier. An ANN for fault involving earth, other ANN for fault not involving earth constitutes a classifier. For classifier ANN design the same procedure, employed as that for Fault. The designed network takes the sets of six inputs 3- $\phi$ current and voltage values scaled corresponding their pre-fault values as inputs. In order to achieve the task of classification by means of ANN training, all fault types with varying severity by changing fault impedance have been simulated on all the three phases at every $15 \mathrm{Km}$ on a $180 \mathrm{Km}$ long transmission line. Both fault location and impedance are changed. Both the ANN has 3 layers, with varying number of neuron in hidden layer. For ANN-I the outputs are either a 0 or 1 corresponding to the L-G or L-L-G fault respectively for the line under consideration. Similarly, the output for ANN-II is either 0 or1 depending upon the type of fault, for L-L fault the output will be 0 or for L-L-L fault the output will be 1. Details of both the ANN for fault classifier designs are shown in table 1 . 


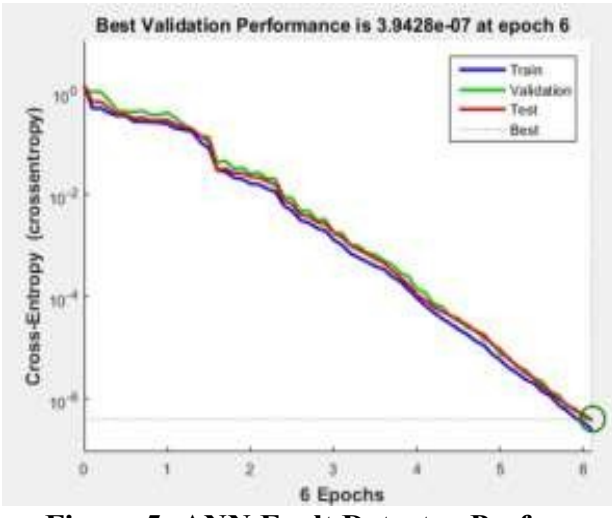

Figure 5: ANN Fault Detector Performances

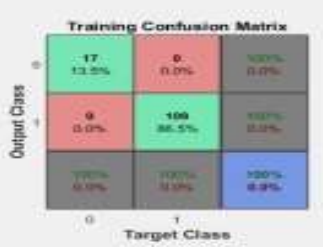

Target Class

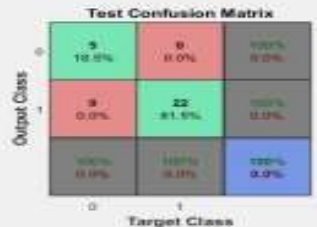

Davoet ciass:

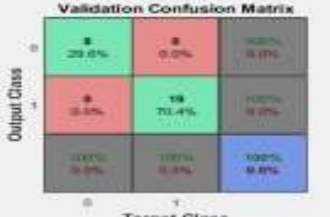

Target class

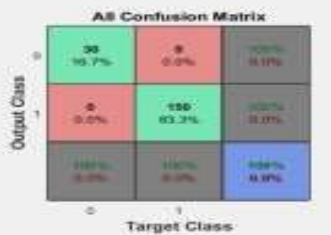

Figure 6: Confusion Matrices of Training, Validation \& Testing Phases of the ANN Fault Detector
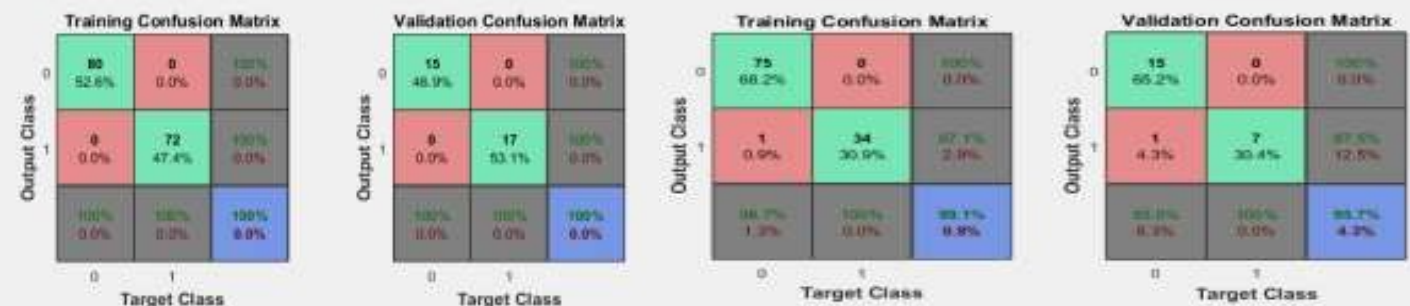

Test Confusion Matrix
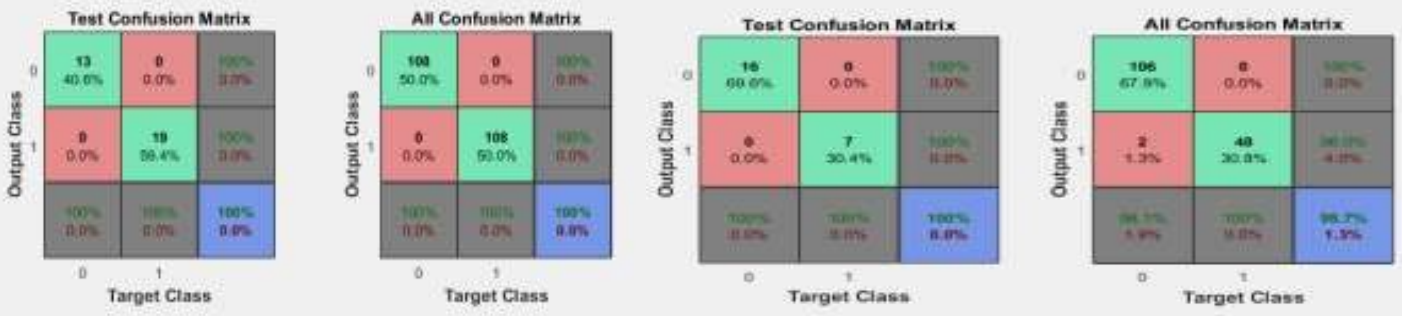

Figure 7(b): Confusion Matrices of

Figure 7(a): Confusion Matrices of Training, Validation \& Testing Phases of the ANN Fault Classifier (Faults involving Earth)

Training, Validation \& Testing Phases of the ANN Fault Classifier (Faults involving Earth)

\begin{tabular}{|l|l|l|}
\hline \multicolumn{1}{|c|}{ Fault Type } & $\begin{array}{l}\text { Neurons per } \\
\text { layer }\end{array}$ & $\begin{array}{l}\text { Maximum } \\
\text { Error }\end{array}$ \\
\hline $\begin{array}{l}\text { Fault involving earth } \\
\text { (ANN-I) }\end{array}$ & $6-12-1$ & $6.6079 \mathrm{e}-07$ \\
\hline $\begin{array}{l}\text { Fault not involving } \\
\text { earth(ANN-II) }\end{array}$ & $6-10-1$ & 0.0079961 \\
\hline
\end{tabular}

Table 1: Fault Classifier Details

\begin{tabular}{|l|l|l|}
\hline Fault Type & Neurons per layer & $\begin{array}{l}\text { Regression } \\
\text { coefficient(r) }\end{array}$ \\
\hline L-G & $6-16-1$ & 0.94756 \\
\hline L-L-G & $6-16-1$ & 0.93292 \\
\hline L-L & $6-18-1$ & 0.93262 \\
\hline L-L-L & $6-11-1$ & 0.99216 \\
\hline
\end{tabular}

Table 2: Fault Location Details

The operation of trained ANN is tested by confusion matrix. Figure.7 (a) and 7(b) shows proficiency of trained ANN as its capability to classify fault type. It is observed that the ANN can distinguish among all type faults on transmission lines.

\section{ANN Design for Fault Location}

As the occurrence of fault can now be detected and it can also be classified in various types of faults, the final task is to accurately determine the location of fault from end. Four different ANN have been developed for each fault type, for identifying the location. Several faults have been simulated on the transmission line model to train the neural network. Fault is simulated after every $3 \mathrm{~km}$ on all the three phases. Fault distance and the resistance are both varied.60 patterns of fault for each of the three phases with each of the 3 different fault resistances have been simulated. Voltage and current samples are given as input to ANN in all of the above cases and the output in all the cases is distance of fault from the receiving end of the transmission line. 
All the fault locator has 3 layers with different neuron in hidden layer. The neurons in each layer are determined by try and error method. Details of network for each type of fault can be found in table 2 .

A separate data set was created to investigate the performance of the developed ANN-based fault locators. The total of 10 test dataset was generated and scaled accordingly for L-G faults, L-L-G faults and L-L faults. The fault resistance was taken as $5 \mathrm{Ohms}$, which was not a part of the training set. This implies ANN's ability to adapt on reception of new data and generalize the same in new class. Fault location was varied from 0 to 180 . Table 3 shows the results of the ANN-based fault locators output.

For ease of analysis, the error in fault location $(\mathrm{km})$ is evaluated using the following mathematical relationship Error $(\mathrm{Km})=[\mathrm{ANN}$ output - Target $]$

ANN output is the distance specified by the fault locator in $\mathrm{km}$ and Target is the actual location where the fault occurred in the transmission line in $\mathrm{km}$ for the considered model. More so, the performance of the fault locators are evaluated using the percentage error expressed mathematically as: [1]

$\%$ Error $=($ Error $/$ Line length $($ in Km $)) \times 100$

\begin{tabular}{|l|l|l|l|}
\hline Type of Fault & Actual Distance & Distance Predicted by ANN & \% Error \\
\hline A-G & 30 & 31.44 & 0.8 \\
\hline B-G & 60 & 61.94 & 1.077 \\
\hline C-G & 120 & 121.31 & 0.727 \\
\hline A-B-G & 30 & 32.04 & 1.13 \\
\hline B-C-G & 60 & 63.84 & 2.13 \\
\hline C-A-G & 120 & 117.42 & -1.433 \\
\hline A-B & 30 & 32.24 & 1.24 \\
\hline B-C & 60 & 61.89 & 1.05 \\
\hline C-A & 120 & 121.97 & 1.094 \\
\hline A-B-C & 120 & 120.96 & 0.544 \\
\hline
\end{tabular}

Table 3: ANN-based Fault Locators Output

\section{Conclusion}

Detection, classification and location of the fault in 3- $\phi$ transmission lines is analysed using ANN. The developed systems utilized inputs as values of instantaneous values of currents and voltages which were processed for detection, fault classification and fault location. The inherent capability of pattern classification and recognition of ANN is utilized in the schemes. ANNs for different application were developed using multilayer perceptron feedforward neural network with backpropagation algorithm. The proposed method is fast, adaptive and accurate due to the application of ANN. Architecture of ANN is found to be accurate, reliable and effective for the problem of detection, classification and location of the faults.

\section{References}

1. Shreya Upadhyay, Dr. S.R. Kapoor, Rajni Choudhary. 2018. Fault Classification and Detection in Transmission Lines using ANN in proceedings of ICIRCA, IEEE 2018, pp. 1029-1034

2. Hagh, M.T.; Razi, K ;Taghizadeh, H. 2007. Fault classification and location of power transmission lines using artificial neural network," in proceedings of International Conference on Power Engineering $1109-1114$.

3. A.S. Neethu and T.S. Angel .2017. Smart Fault Location and Fault Classification in Transmission Line in proceedings of ICSTM, IEEE 2 - 4pp.339-343.

4. Preeti Gupta, R. N. Mahanty. 2016. Artificial Neural Network based Fault Classifier and Locator for Transmission Line Protection" in proceedings of IOSR-JEEE, Volume 11, Issue 1 Ver. I, Jan - Feb. 2016, PP 4153

5. Teklic, L.; Filipovic-Grcic, 8. ; Pavicic, I. 2013. Artificial neural network approach for locating faults in power transmission system," in proceedings of EUROCON, IEEE, Zagreb, pp. 1425-1430

6. Raoofat, M.; Mahmoodian, A.; Abunasri, A. 2015. Fault location in transmission lines using neural network and wavelet transform in proceedings of International Congress on Electric Industry Automation, Feb.

2015, pp. 1-6.

7. Y. Aslan, Y. EmreYa_. 2016. an ANN Based Fault Location for Medium Voltage Distribution Lines with "Remote-end Source" in international symposium on fundamental of electrical engineering, university politehnica of Bucharest, Romania, IEEE june 30- july 2, 2016.

8. M. Jamil, S. K. Sharma, and R. Singh. 2015.Fault detection and classification in electrical power transmission system using artificial neural network Springer Open Journal, 4:334 DOI 10.1186/s40064-015-1080-x, 2015

9. D. Thukaram, U. J. Shenoy, and H. Ashagesstha, 2006. Neural network approach for fault location in unbalanced distribution network with limited measurement, in Proc. IEEE Power India Conf., New Delhi, India, pp.

493-500, Apr. 2006. 